\begin{tabular}{|c|l|}
\hline Title & Morphological characterization of the diblock copolymer problem with topol ogical computation \\
\hline Author(s) & Teramoto, Takashi; Nishiura, Y asumasa \\
\hline Citation & $\begin{array}{l}\text { Japan Journal of Industrial and A pplied Mathematics, 27(2), 175-190 } \\
\text { https://doi.org/10.1007/313160-010-0014-9 }\end{array}$ \\
\hline Issue Date & 2010-09 \\
\hline Doc URL & http://hdl.handle.net/2115/44297 \\
\hline Rights & The original publication is available at www.springerlink.com \\
\hline Type & article (author version) \\
\hline File Information & JJA M27-2_175-190.pdf \\
\hline
\end{tabular}

Instructions for use 


\title{
Morphological Characterization of the Diblock Copolymer Problem with Topological Computation
}

\author{
Takashi Teramoto · Yasumasa Nishiura
}

Received: date / Accepted: date

\begin{abstract}
Our subject is the diblock copolymer problem in a three-dimensional space. Using numerical simulations, the double gyroid and orthorhombic morphologies are obtained as energy minimizers. By investigating the geometric properties of these bicontinuous morphologies, we demonstrate the underlying mechanism affecting the triply periodic energy minimizers in terms of a balanced scaling law. We also apply computational homology to their characterization during the dynamics of morphology transition. Our topological approaches detect the morphology of transient perforated layers as they transition from layers to cylinders, and the $t^{-1}$ law of the Betti number in the phase ordering process.
\end{abstract}

Keywords double gyroid · microphase separation · the Betti number

\section{Introduction to microphase separated morphology}

Much more than a mere means of expression, mathematical description used to be assimilated into physical laws themselves, including the definitions of fundamental concepts. However, in recent years there has been a disassociation between materials science and mathematics. It is our belief that much can be learned by merging the viewpoints of these respective disciplines. It is relatively easy to combine diverse disciplines using computer graphics as an interface, similar to how a "surfactant" can tentatively contribute to fusion. Such an approach has often been taken for interdisciplinary work in computational science, but on a microscopic level the disciplines remain separated, despite the appearance of being uniformly mixed. This is analogous to "microphase separation" which is the subject of this paper.

Microphase separation is driven by a chemical incompatibility between different blocks that compose block copolymer molecules. In a diblock copolymer melt, the

Takashi Teramoto

Chitose Institute of Science and Technology, Chitose 066-8655, Japan

E-mail: teramoto@photon.chitose.ac.jp

Yasumasa Nishiura

Research Institute of Electronic Science, Hokkaido University, Sapporo 001-0020, Japan 

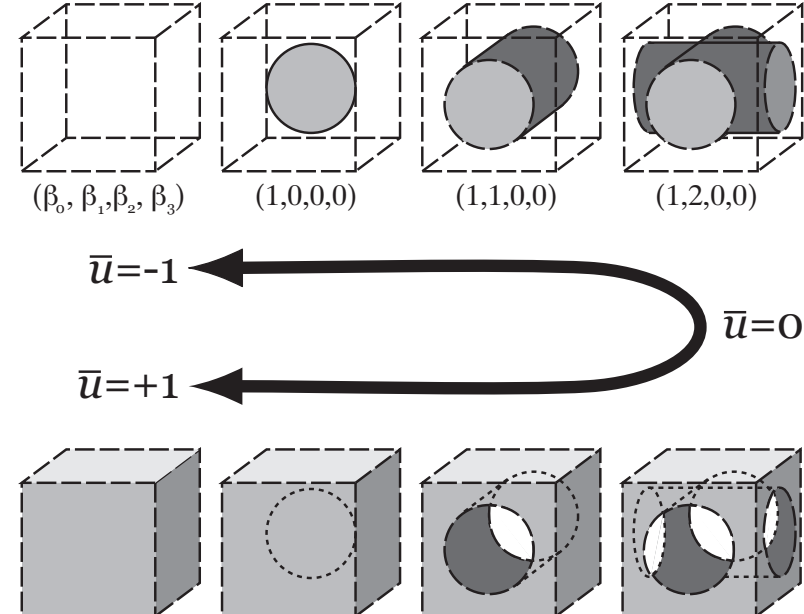

$(1,3,3,1)$

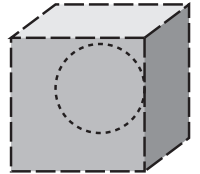

$(1,3,3,0)$

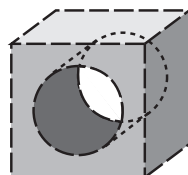

$(1,3,2,0)$

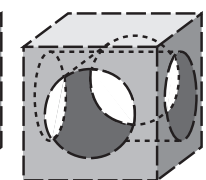

$(1,3,1,0)$

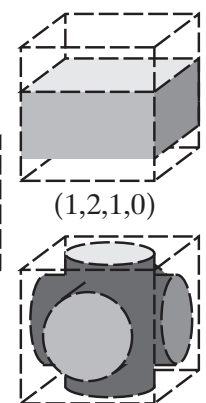

$(1,3,0,0)$

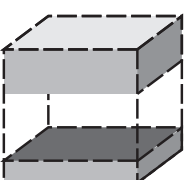

$(1,2,1,0)$

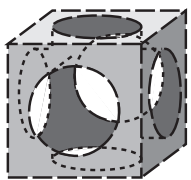

$(1,3,0,0)$

Fig. 1 Schematic image of sequences of the periodic morphologies in a three-dimensional space, depending on the diblock composition $\bar{u}$. The complexity of the morphology arises for nearly symmetric diblocks of $\bar{u} \simeq 0$. Three-dimensional morphologies are characterized by using the topological invariants, the set of the Betti numbers $\beta_{k}$ for $k=0, \cdots 3$. The Betti numbers change gradually by adding one along the line-up, following the incremental rule. The details will be provided in later sections.

final equilibrium state then tends to contain periodic structures such as layers, cylinders, spheres and double gyroid bicontinuous morphologies at a molecular length scale $(10 \sim 100 \mathrm{~nm})[5,15]$. Why do such morphologies appear in the mixtures ? One can guess along the following lines: the dominant factor in determining the morphology is the area-minimization of the interface region enclosed by which has a fixed volume. Surfaces that satisfy this mathematical condition are said to have a constant mean curvature $(\mathrm{CMC})$. The familiar triply periodic cubic geometries, such as primitive $(\mathrm{P})$, diamond (D), and gyroid (G) surfaces, form a representative class of CMC surfaces $[14,27]$. It is well established both experimentally and numerically that the diblock copolymer problem can generate complex three-dimensional morphologies. Quantitatively characterizing the geometry of these complex morphologies is a long-standing challenge. Algebraic topology is a classical mathematical tool for the global analysis of nonlinear systems and functions, and it provides very basic topological (geometric) information about the patterns, including the number of components (pieces) and the number of holes. When used for three-dimensional morphologies, it also provides the number of voids. Computationally efficient algorithms and software for homology have been developed, allowing us to compute the homology of cubical sets and maps. Thus, complex structures obtained from experimental observations and numerical simulations can be quantified using a coherent set of mathematical techniques $[10,16]$. 


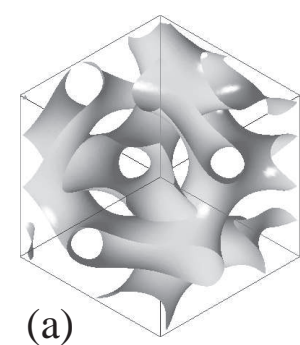

(a)

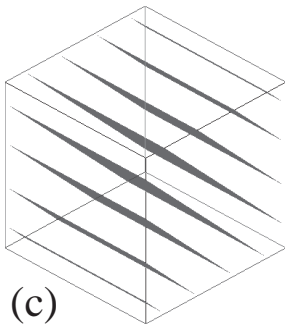

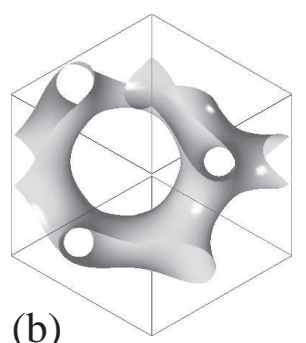

(b)

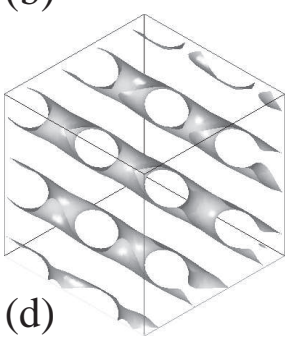

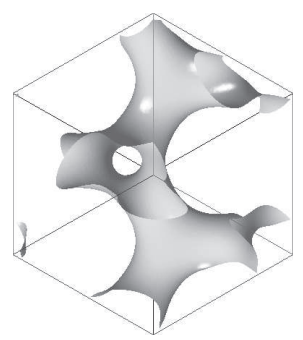

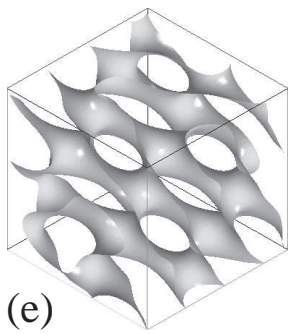

Fig. 2 The views from the [111]-direction of isosurfaces obtained as the energy minimizers of the diblock copolymer problem at $\bar{u}=0.10$ : (a) Double gyroid; (b) a pair of gyroid singlenetworks forming a chiral-network; (c) Layers (the layer interval is $2^{-3 / 2} l$ ); (d) Perforated Layers; and (e) Orthorhombic.

The contribution of this paper is twofold. First, we reveal the underlying mechanism affecting the double gyroid (DG) morphology of the diblock copolymer problem, by investigating the curved surface based on its differential geometry. Second, we demonstrate specific applications of computational homology that characterize the topological changes during the morphology transition in three-dimensional spaces. This paper is organized as follows. In Sections 2 and 3, we introduce the diblock copolymer problem, and then we carefully demonstrate the geometric properties of the energy minimizers that are obtained numerically in three-dimensional space. In Section 4 , we employ computational homology to investigate the topological properties of the minimizers and the morphology transition between them. Finally, a summary is provided in Section 5.

\section{Energy minimizers of the diblock copolymer problem}

Active research into block copolymers has been occurring since relatively early days in Japan [1], including the group in the Faculty of Engineering at Kyoto University. In fact, in the 1980s it was experimentally demonstrated that the intensity of scattering in a molten liquid state exhibits a wavenumber peak characteristic of the mesoscale. By using the Fourier transformation of the concentration fluctuation observed directly in scattering experiments, Ohta and Kawasaki proposed a phenomenological model based on the Landau-type free energy of macrophase separation[3,22]. They simply incorporated a Coulomb-type long-range restraining effect which competes with the interaction of short-range attraction by diffusion. Apparently, a few applied mathematicians were able to realize various energy-minimized conditions, by adding a non-local effect to a one-variable scalar Allen-Cahn equation model using integral conditions [19,20]. When 


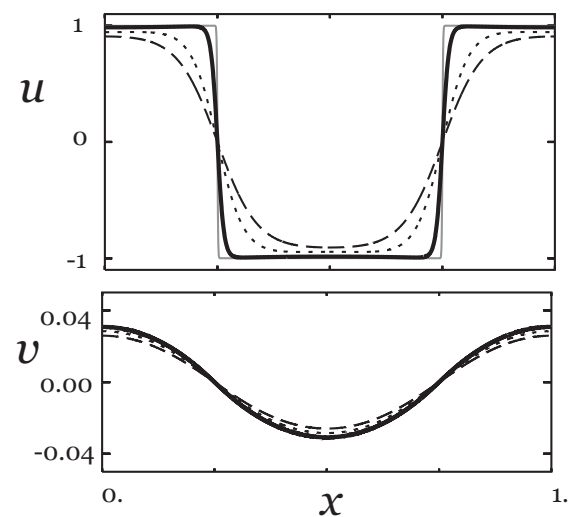

(a)

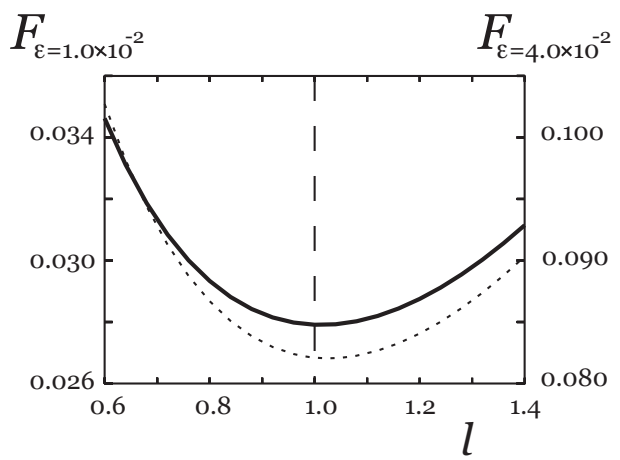

(b)

Fig. 3 (a) The $\epsilon$-dependence of profiles of the one-dimensional minimizer. The chemical potential $v$ satisfies $-v_{x x}=u-\bar{u}, v_{x}(0)=v_{x}(1)=0$ and $\int_{0}^{1} v \mathrm{~d} x=0$. The $\epsilon$ are chosen as follows; broken: $6.0 \times 10^{-2}$, dotted: $4.0 \times 10^{-2}$, solid: $1.0 \times 10^{-2}$, gray: $1.0 \times 10^{-3}$. (b) The minimization of $F_{\epsilon, \sigma}$ with respect to $l$ when $\tilde{\sigma}=2^{13 / 2}\left(1-\bar{u}^{2}\right)^{-2}$. The $\epsilon$ are chosen as follows; solid: $1.0 \times 10^{-2}$, dotted: $4.0 \times 10^{-2}$.

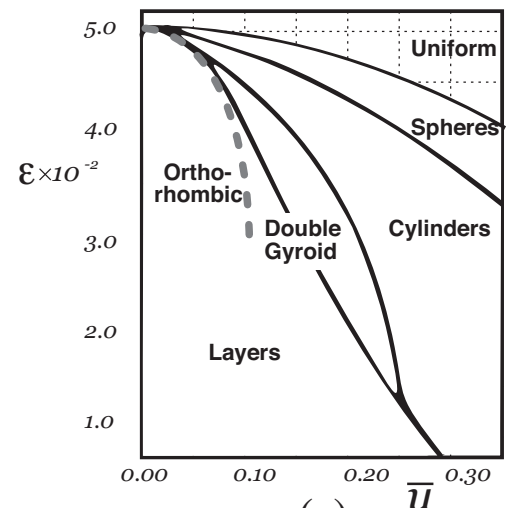

(a)

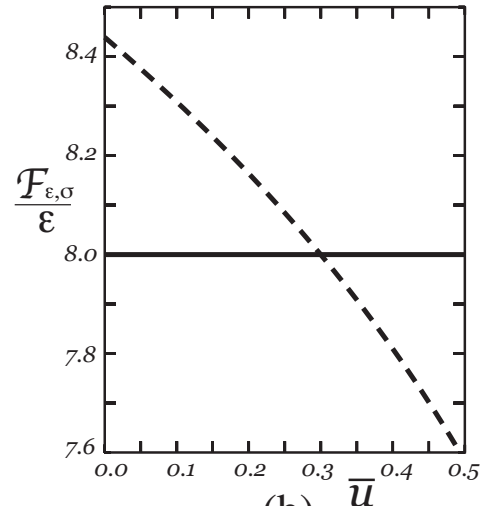

(b)

Fig. 4 (a) $(\bar{u}, \epsilon)$-phase diagram of global minimizers, including the hexagonal cylinders, bodycentered cubic spheres and the uniform state, when $\tilde{\sigma}=2^{11}\left(1-\bar{u}^{2}\right)^{-2}$. The broken gray curve shows the border between the double gyroid-orthorhombic (DG-OR) phases. In approaching $\bar{u}=0.00$, the $F_{\epsilon, \sigma}$ value for OR morphology becomes smaller than that of DG. (b) The transition point between layers and cylinders in the singular limit of $\epsilon \rightarrow 0$ are computed as $\bar{u} \approx 0.2997$. Solid and broken lines indicate the $F_{\epsilon, \sigma} / \epsilon$ values obtained from (2) for layers and (5) for cylinders, respectively.

the scaling rule of the domain size was exactly solved by Nishiura and Ohnishi, this model was mathematically re-formulated, a fact which had great significance. The free energy functional, $F_{\epsilon, \sigma}$, consists of the following three parts $\left(F_{\epsilon, \sigma}=E_{1}+E_{2}+E_{3}\right)$, 
and its minimum value will produce a thermal equilibrium state:

$$
F_{\epsilon, \sigma}(u)=\int_{\Omega}\left(\frac{\epsilon^{2}}{2}|\nabla u|^{2}+W(u)+\frac{\sigma}{2}\left|(-\triangle)^{-1 / 2}(u-\bar{u})\right|^{2}\right) \mathrm{d} \boldsymbol{r},
$$

where $\bar{u}=|\Omega|^{-1} \int_{\Omega} u \mathrm{~d} \boldsymbol{r}$ is the space average of the local concentration (diblock composition), $u(\boldsymbol{r})$. The phenomenological parameters, $\epsilon$ and $\sigma$, are the interface thickness and the strength of the chemical bond, respectively; both are derived from the FloryHuggins interaction parameter and from microscopic quantities, such as the degree of monomer polymerizations. The double well potential, $W(u)=\left(u^{2}-1\right)^{2} / 4$, occurring in the second term $E_{2}$, separates into $u \sim+1$ and $u \sim-1$, relative to two stable concentrations of the local concentration $u(\boldsymbol{r})$. The first term acts to decrease the area of the interfacial region, and the third term acts to promote formation of the interface; this situation leads to the the competition between short-range activation and longrange restraint. With a system as described here, even though some details may differ, a pattern with an equally spaced appearance on the mesoscale will result. Choksi and Ren re-derive the functional (1) using self-consistent mean field theory, which connects those phenomenological parameters to microscopic material parameters [8].

In this study, we focus on the characterization of the global minimizers of order $O(1)$, i.e., those with a periodic structure in space where $\Omega$ is the unit cell, for the diblock copolymer problem of $\min \left\{F_{\epsilon, \sigma} / \epsilon:|\Omega|^{-1} \int_{\Omega} u \mathrm{~d} \boldsymbol{r}=\bar{u}\right\}$. In the limit $\epsilon \rightarrow 0$, all three terms of order $O(\epsilon)$ tend to equally contribute to $F_{\epsilon, \sigma}$. Assuming a sharp interface interpolation for the stacked layers, we can replace $\epsilon^{2} \int_{\Omega}|\nabla u|^{2} \mathrm{~d} \boldsymbol{r} / 2+\int_{\Omega} W(u) \mathrm{d} \boldsymbol{r}$ by $\epsilon \int_{\Omega}|\nabla u| \mathrm{d} \boldsymbol{r}$. As a result, $E_{1}+E_{2}$ tends to about $\epsilon$ times the total area of the interface region of $O(1)$. The $\Gamma$-convergence approach gives convergence of minimum energy values and minimizers. We refer the readers to Chapter $6[6]$ for its properties. The $\Gamma$-limit of a rescaled functional $F_{\epsilon, \sigma} / \epsilon$ for the $\nu$-mode layer solution $u_{\nu}$ in $\Omega=[0,1]$ can be written as:

$$
\Gamma-\lim _{\epsilon \rightarrow 0^{+}} \frac{F_{\epsilon, \sigma}\left(u_{\nu}\right)}{\epsilon}=c \nu+\frac{\left(1-\bar{u}^{2}\right)^{2}}{24 \nu^{2}} \tilde{\sigma}, \quad \text { if } u \in P C(\Omega),
$$

where $c=\int_{-1}^{1} \sqrt{2 W(u)} \mathrm{d} u$ and $P C(\Omega)$ is the space of piecewise constant function on $\Omega$. Here, we introduce the scaled parameter by $\tilde{\sigma}=\sigma / \epsilon$. A complete study is performed by Ren and Wei [23], in which they adapted to the general case giving a condition called BV-space. In this paper, we numerically confirm that the transition of the phase separation phenomenon to the minimum problem can be understood on a scale $O(1)$.

By varying (2) with respect to $\nu$ in $\Omega=[0,1]$, the 2-mode type solution can be characterized as the global minimizer if $\tilde{\sigma}=2^{13 / 2}\left(1-\bar{u}^{2}\right)^{-2}$. Each term of $F_{\epsilon, \sigma}$ therefore becomes equally balanced, as $F_{\epsilon, \sigma}\left(u_{2}\right) / \epsilon=4 \sqrt{2} / 3+2 \sqrt{2} / 3=2 \sqrt{2}$. This is verified numerically by computing the $F_{\epsilon, \sigma}$ value as $\left(E_{1}, E_{2}, E_{3}\right)=\left(9.44 \times 10^{-3}, 9.41 \times\right.$ $\left.10^{-3}, 9.41 \times 10^{-3}\right)$ for $(\epsilon, \bar{u})=\left(1.0 \times 10^{-3}, 0.00\right)$. Figure 3 graphs the $F_{\epsilon, \sigma}$ values for the 2-mode type minimizers, for which the layer interval is $l(\Omega=[0, l))$. For small $\epsilon$, the global minimizer is obtained if the optimal system size $l=1$, while for large $\epsilon$, the optimal system size $l$ is slightly larger than unity.

Hereafter, $\Omega \subset \boldsymbol{R}^{3}$ is taken to be the cube $[0, l)^{3}$, subject to the periodic boundary condition. In order to realize the one unit of triply periodic cubic geometry, we apply $\tilde{\sigma}=2^{11}\left(1-\bar{u}^{2}\right)^{-2}$, in which the L structure (the layer interval is $2^{-3 / 2} l$ ) gives the period of the global minimizer as $\epsilon \rightarrow 0$. This justification for this depends on whether the three-dimensional structures organize as observable phenomena, and not on whether 
the theory was used correctly from a mathematical standpoint. Nevertheless, the theoretical result described above leads us to confirm the DG morphology and the other CMC type morphologies in the gradient system of (1) $[25,26]$.

Figure 4(a) shows the $(\bar{u}, \epsilon)$-phase diagram of global minimizers, where the scaled parameter is fixed at $\tilde{\sigma}=2^{11}\left(1-\bar{u}^{2}\right)^{-2}$. Here, we numerically compare the energy density, $\mathcal{F}_{\epsilon, \sigma}=|\Omega|^{-1} F_{\epsilon, \sigma}=\mathcal{E}_{1}+\mathcal{E}_{2}+\mathcal{E}_{3}$, among the candidate CMC type structures. Note that we do not take into account non-CMC type structures such as the ring patterns found recently by Kang and Ren [17]. In the phase diagram, the double gyroid morphology becomes a global minimizer in the intermediate range of the parameter $\epsilon$, along the layers-cylinders phase boundary. We compare the contributions from each of the three terms of the energy density, $\mathcal{F}_{\epsilon, \sigma}$, for the lower L-DG-C triple point vicinity $(\bar{u}, \epsilon) \approx\left(0.27,1.0 \times 10^{-2}\right)$. For the layers $(l \simeq 1.04),\left(\mathcal{E}_{1}, \mathcal{E}_{2}, \mathcal{E}_{3}\right) / \epsilon \approx(2.50,2.74,2.46)$ and $\mathcal{F}_{\epsilon, \sigma} / \epsilon=7.70$. For the cylinders $(l \simeq 1.01)$ and the double gyroid $(l \simeq 0.89)$, the three contributions are $(2.42,2.88,2.41)$ and $(2.46,2.81,2.44)$, respectively. Both of them total to 7.71 , and they balance at $O(1)$ from equilibrium scaling. Because the surface areas of the interface $S(\Gamma(\bar{u}))$ are 5.44,5.67, and 5.66, currently, the relational expression expected at the limit, $\lim _{\epsilon \rightarrow 0^{+}}\left(\mathcal{E}_{1}+\mathcal{E}_{2}\right) / \epsilon \simeq(2 \sqrt{2} / 3) S(\Gamma(\bar{u}))$ holds at $O(1)$. We note the separating surface $\Gamma(\bar{u})=\{\boldsymbol{r} \in \Omega \mid u(\boldsymbol{r})=\bar{u}\}$, and two domains $u^{-}=$ $\{\boldsymbol{r} \in \Omega \mid u(\boldsymbol{r})<\bar{u}\}$ and $u^{+}=\{\boldsymbol{r} \in \Omega \mid u(\boldsymbol{r})>\bar{u}\}$.

By minimizing $F_{\epsilon, \sigma}$ numerically, we are looking for cubic morphology of order $O(1)$, that is, it has a periodic structure in space with $\Omega$ being its unit domain. Here we perform numerical simulations of the following Euler-Lagrange equation for a gradient system that ensures that the Lyapunov function $F_{\epsilon, \sigma}$ will monotonically decrease over time, while conserving the average $\bar{u}$ :

$$
\frac{\partial u}{\partial t}=\Delta\left(\frac{\delta F_{\epsilon, \sigma}}{\delta u}\right)=\Delta\left(-\epsilon^{2} \Delta u+\frac{\partial W(u)}{\partial u}\right)-\sigma(u-\bar{u}) .
$$

In order to solve the equation above in a gradient-stable manner [11], we apply the semi-implicit method to the Cahn-Hilliard equation with the nonlocal term. We have used the following semi-implicit scheme:

$$
\frac{u_{i j k}^{n+1}-u_{i j k}^{n}}{h}=-\epsilon^{2} \triangle_{d}^{2} u_{i j k}^{n+1}+\triangle_{d}\left(\frac{\partial W\left(u_{i j k}^{n}\right)}{\partial u}\right)-\sigma\left(u_{i j k}^{n+1}-\bar{u}\right),
$$

where $u_{i j k}^{n}$ denotes the discrete approximation of $u(i d, j d, k d, n h), h$ is the time step, and the space discretization is $d$. In this manner, the linear equations $M^{-1}\left(I+h \epsilon^{2} \Lambda_{d}^{2}+\right.$ $\sigma) u_{i j k}^{n+1}=M^{-1}\left[u_{i j k}^{n}-h \Lambda_{d}\left(\partial W\left(u_{i j k}^{n}\right) / \partial u\right)+\sigma \bar{u}\right]$ are obtained and solved by defining the preconditioner to be $M=I+h \epsilon^{2} \Lambda_{d}^{2}+\sigma$, where the matrix $\Lambda_{d}$ is the discrete Laplacian and the standard centered difference approximation of the Laplacian is used. This preconditioned system can be quickly solved by using a combination of the FFT method and conjugate gradient method. By virtue of the use of Fourier series representation $u \boldsymbol{n}\left(\boldsymbol{n} \in \boldsymbol{Z}^{3}\right)$, the nonlocal term is computed by $E_{3}=(\sigma / 2) \sum_{\boldsymbol{n} \in \boldsymbol{Z}^{3}}|u \boldsymbol{n}|^{2} / \lambda \boldsymbol{n}$, where $\lambda \boldsymbol{n}$ denotes the $n$-th eigenvalues of $(-\triangle)$ and the sum over excludes the $\boldsymbol{n}=0$ term.

\section{Geometric properties of global minimizers}

In this section, we investigate the measures of the level set $\Gamma(\bar{u})$ obtained in the previous section. Table I shows the border region whether the surface area $\Gamma(\bar{u})$ of the 
Table 1 The $\bar{u}$-dependence of surface area $S(\Gamma(\bar{u}))$ and the total mean curvature $\left(\int_{\Gamma(\bar{u})} H \mathrm{~d} S\right)$ over the DG-OR transition region

\begin{tabular}{ccccccccc}
\hline \multicolumn{1}{c}{$S(\Gamma(\bar{u}))$} & \multicolumn{4}{c}{$\int_{\Gamma(\bar{u})} H \mathrm{~d} S$} \\
$\bar{u}$ & $\epsilon=1.0 \times 10^{-2}$ & $\epsilon=4.0 \times 10^{-2}$ & \multicolumn{4}{c}{$\epsilon=1.0 \times 10^{-2}$} & $\epsilon=4.0 \times 10^{-2}$ \\
& $\mathrm{DG}$ & OR & $\mathrm{DG}$ & OR & DG & OR & DG & OR \\
\hline 0.00 & 5.570 & $\mathbf{5 . 3 3 6}$ & 5.353 & $\mathbf{5 . 2 9 3}$ & 8.581 & $\mathbf{3 . 3 3 1}$ & 9.878 & $\mathbf{0 . 1 9 7}$ \\
0.05 & 5.497 & $\mathbf{5 . 3 0 8}$ & 5.306 & $\mathbf{5 . 2 7 0}$ & 9.310 & $\mathbf{4 . 3 7 1}$ & 10.097 & $\mathbf{4 . 8 2 1}$ \\
0.08 & 5.450 & $\mathbf{5 . 2 8 7}$ & 5.275 & $\mathbf{5 . 2 5 7}$ & 9.687 & $\mathbf{4 . 9 3 4}$ & 10.298 & $\mathbf{5 . 7 9 5}$ \\
0.09 & 5.435 & $\mathbf{5 . 2 7 9}$ & 5.261 & $\mathbf{5 . 2 5 3}$ & 9.814 & $\mathbf{5 . 1 2 4}$ & 10.178 & $\mathbf{6 . 0 7 5}$ \\
0.10 & 5.420 & $\mathbf{5 . 2 7 2}$ & 5.250 & $\mathbf{5 . 2 4 8}$ & 9.944 & $\mathbf{5 . 3 1 4}$ & 10.238 & $\mathbf{6 . 3 2 6}$ \\
0.11 & 5.405 & $\mathbf{5 . 2 6 4}$ & 5.239 & $\mathbf{5 . 2 4 2}$ & 10.075 & $\mathbf{5 . 4 7 7}$ & 10.301 & $\mathbf{6 . 5 5 8}$ \\
0.12 & 5.389 & $\mathbf{5 . 2 5 6}$ & 5.228 & $\mathbf{5 . 2 3 7}$ & 10.258 & $\mathbf{5 . 6 7 5}$ & 10.367 & $\mathbf{6 . 7 7 4}$ \\
0.15 & 5.339 & $\mathbf{5 . 2 3 0}$ & 5.192 & $\mathbf{5 . 2 1 9}$ & 10.731 & $\mathbf{6 . 2 5 5}$ & 10.570 & $\mathbf{7 . 5 3 4}$ \\
0.20 & 5.249 & $\mathbf{5 . 1 8 2}$ & 5.128 & $\mathbf{5 . 1 8 8}$ & 11.326 & $\mathbf{7 . 2 2 0}$ & 10.916 & $\mathbf{8 . 7 9 2}$ \\
\hline
\end{tabular}

Table 2 The Betti number set and space group symmetry for the triply periodic morphologies, such as primitive, gyroid, diamond, and orthorhombic, appear in diblock copolymer problems.

\begin{tabular}{|c|c|c|c|c|c|c|}
\hline & \multicolumn{3}{|c|}{ single network } & \multicolumn{3}{|c|}{ double network } \\
\hline & $\beta_{k}\left(X^{-}\right)$ & $\beta_{k}\left(X^{+}\right)$ & symmetry & $\beta_{k}\left(X^{-}\right)$ & $\beta_{k}\left(X^{+}\right)$ & symmetry \\
\hline $\mathrm{P}$ & $(1,3,0,0)$ & $(1,3,0,0)$ & Pm̄̄m & $(2,6,0,0)$ & $(1,6,1,0)$ & $\operatorname{Im} \overline{3} \mathrm{~m}$ \\
\hline G & $(1,5,0,0)$ & $(1,5,0,0)$ & $\mathrm{I} 4_{1} 32$ & $(2,10,0,0)$ & $(1,10,1,0)$ & $\mathrm{Ia} \overline{3} \mathrm{~d}$ \\
\hline $\mathrm{D}$ & $(1,9,0,0)$ & $(1,9,0,0)$ & $\mathrm{Fd} \overline{3} \mathrm{~m}$ & $(2,18,0,0)$ & $(1,18,1,0)$ & Pn̄̄m \\
\hline OR & $(1,13,0,0)$ & $(1,13,0,0)$ & Fddd & & & \\
\hline
\end{tabular}

orthorhombic (OR) morphology is smaller than that of DG; this region is around $\bar{u}=0.10$ for $\epsilon=4.0 \times 10^{-2}$, and is consistent with the DG-OR border line in Fig.4(a). In particular for $\bar{u}=0.00$, the mean curvature of the interface $\Gamma(\bar{u})$ of OR approaches zero, while that of DG is 1.84 . In addition, when $\epsilon$ is large, the interfacial energy $E_{1}+E_{2}$ dominates the total energy $F_{\epsilon, \sigma}$. Therefore, the interface $\Gamma(\bar{u})$ of our system can be well approximated by DG with CMC.

In [26], we used the symbol "R" instead of OR, however, OR may be appropriate for the orthorhombic form of the Fddd symmetry [4]. We estimate that $80 \%$ of the total surface has zero or negative Gaussian curvature $K$ for $\epsilon=4.0 \times 10^{-2}$ (67\% for $\left.\epsilon=1.0 \times 10^{-2}\right)$. In other words, the interface $\Gamma(\bar{u})$ of OR has numerous saddle-shaped areas. Figure 2 depicts the unit cell investigated here. The Euler characteristic for OR is $\chi(\Gamma(\bar{u}))=(2 \pi)^{-1} \int_{\Gamma(\bar{u})} K \mathrm{~d} S=-24$, while that for DG is $-16(=-8 \times 2)$. In this connection, each single network of DG shown in Fig.2 has a diblock composition of $22.5 \%$, a surface area $\Gamma(\bar{u})$ of 2.63 , and a mean curvature of about 1.94 . The surface area $\Gamma(\bar{u})$ of 2-period $\mathrm{G}$ is 6.24 , and the mean curvature is nearly zero when $(\epsilon, \bar{u})=$ $\left(4.0 \times 10^{-2}, 0.10\right)$. The results in Table I indicate the existence of a $\bar{u}$-family of OR surfaces. Here the mean and Gaussian curvatures of the level set $\Gamma(\bar{u})$ are computed from the distribution of a scalar field $u$ on a cubic lattice, by using the numerical method developed by Hołyst et al $[2,13]$. In addition, we refer the readers to the reference [24] for their formulas. The accuracy of the computation depends on the space discretization, since the points on $\Gamma(\bar{u})$ are approximated by the interpolation of the cubic lattice points. Here we set $d=2^{-7}$. 
Here we provide an intuitive explanation for the selection of a gyroid instead of other cubic morphologies, why it forms a double network, and why it emerges in a more complicated Ia $\overline{3}$ d-symmetry. Within a unit cube cell, $|\Omega|=1$, we compare the surface area of the layers interface, $2^{5 / 2}$, of the stripe interval $\mathcal{P}=2^{-3 / 2}$, with an ideal cubic phase interface having a constant average curvature. Then, we note that the energy minimization problem can be replaced with the following geometric problem: how to reduce the surface area, replaced by its perimeter as denoted by $\operatorname{Per}\left(u^{-}, \Omega\right)$, of the boundary of the $u^{-}$region inside the region $\Omega$, in relation to its $\Gamma$-limit with $\epsilon \rightarrow 0$ :

$$
\min \left\{\operatorname{Per}(\{u=-1\}, \Omega): u \rightarrow \pm 1,|\Omega|^{-1} \int_{\Omega} u \mathrm{~d} \boldsymbol{r}=\bar{u}, \mathcal{P}=2^{-3 / 2}\right\} .
$$

The periodic morphology emerges as what minimizes the perimeter between the two phases on a scale comparable to a unit cube. Suppose that the diblock composition is simply $50 \%$. Then, the single network has a 4 -mode $(4 \pi)$ structure in a unit cell. In this situation, the perimeter of the gyroid interface is $6.18(=3.09 \times 2)$, that of the primitive is $6.65\left(\approx 2.35 \times 2^{3 / 2}\right)$, and that of the diamond is 7.68 . Each of them is a local minimizer. The perimeter of the gyroid interface is the smallest of the cubic morphologies, but it is rather larger than that of the layer interface $(=4 \sqrt{2})$; therefore, the likelihood of realizing those single networks is low. Next, we consider double networks; for example, with two networks having a composition of $25 \%$, a 2 -mode $(2 \pi)$ structure is formed in a unit cell. The perimeter of the double gyroid interface is $5.46(=2.73 \times 2)$, that of the double primitive is $5.66\left(\approx 2.00 \times 2^{3 / 2}\right)$, and that of the double diamond is 6.72 . Since the surface area of the interface region is a decreasing convex function with respect to the diblock composition, the double network is smaller in either case. Thus, a double gyroid would be more likely than other morphologies to be realized for a thermal equilibrium state, because it can achieve an interface that is smaller than that of a layer. Furthermore, numerical simulations can identify an orthorhombic morphology with Fddd-symmetry. Its $\mathcal{F}_{\epsilon, \sigma}$ value can be smaller and more stable than that of the double gyroid for nearly a symmetric composition. The perimeter for this level set $\Gamma(\bar{u})$ is $\operatorname{Per}\left(u^{-}, \Omega\right) \approx 5.33$ for $\epsilon=1.0 \times 10^{-2}$, which is smaller than $\operatorname{Per}\left(u^{-}, \Omega\right) \approx 5.57$ for the double gyroid. If such an interface with constant mean curvature exists, then its surface area will be even smaller. This is because the morphology at the singular limit would extend the average curvature distribution of its level set $\Gamma(\bar{u})$ according to the free boundary value problem in relation to the chemical potential. The nonlocal term can affect the shape of a stable morphology and deviate from CMC surfaces.

\section{Applications of computational Homology}

In the previous section, we investigated these two geometric measures, the surface area and the curvature of the level set $\Gamma(\bar{u})$, for the three-dimensional morphologies obtained as minimizers in the diblock copolymer problem. In this section we present a new viewpoint for understanding complex dynamics, including morphology transitions. To do this, we incorporate topologically invariant quantities, the so-called homological quantities, into the field of materials science, and use them as a means of observation. We use homology to measure the topology of a structure, more specifically, we compute its Betti numbers. For precise definitions and algorithms for computing homology, see $[10,16]$. 

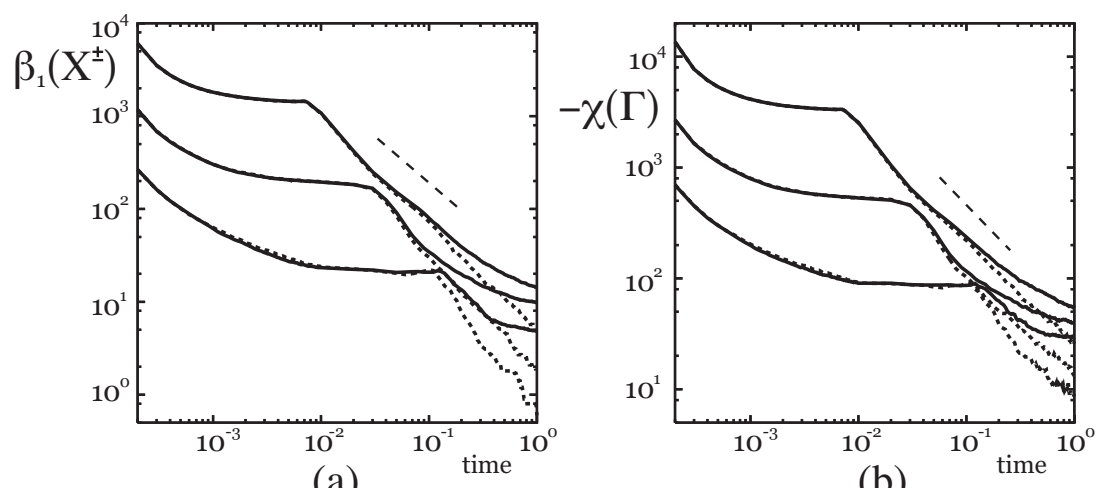

(a)

(b)

Fig. 5 (a) The solid (respectively dotted) lines indicate time evolution of the first Betti number $\beta_{1}\left(X^{1}\right)$ during the micro- (respectively macro-) phase separation with $\sigma=2^{19 / 2} \epsilon$ (respectively $\sigma=0)$. Time evolution of $\beta_{1}\left(X^{ \pm}\right)$looks very similar to that of $-\chi(\Gamma(\bar{u}))$ as shown in (b). The graphs for the different $\epsilon$ values of $1.0 \times 10^{-2}, 2.0 \times 10^{-2}$, and $4.0 \times 10^{-2}$ are drawn from top to bottom in each figure. The dashed line indicates the power law of $t^{-1}$.

To obtain the data structure needed in the computations, the topological space is decomposed into elementary cubes based on rectangular grids. The local concentration $u(\boldsymbol{r})$ is represented by $\left\{u_{i j k} \mid 1 \leq i, j, k \leq N\right\}$. We determine the Betti number $\left\{\beta_{k}\left(X^{ \pm}\right)=\right.$rank $\left.H_{k}\left(X^{ \pm}\right) \mid k=0, \cdots, 3\right\}$ for the cubical sets $X^{ \pm}$divided on the interface $\Gamma(\bar{u})$. The three-dimensional bicontinuous morphologies can be classified in terms of the Betti numbers (Table 2). Notice that these Betti numbers provide more detailed topological information than the Euler characteristic $\chi$ obtained through the GaussBonne theorem, since $\chi$ is also computed as the alternative summation of the Betti numbers by $\chi\left(X^{ \pm}\right)=\sum_{k=0}^{3}(-1)^{k} \beta_{k}\left(X^{ \pm}\right)$. Hereafter, we propose the use of computational homology to quantify the geometry of intriguing and complicated patterns that are produced by morphology transition dynamics.

\subsection{Scaling law of the Betti number during the phase separation process}

If a system becomes unstable in its equilibrium state, generally it undergoes changes to form a new, stable, and ordered phase. This order formation process is called spinodal decomposition $[14,18]$. In this subsection, we perform a topological characterization of the dynamics of phase separation, in which the unstable uniform state $(\bar{u}=0.0)$ is slightly perturbed initially. The numerical results depicted in Fig. 5 are averaged over 20 different initial configurations. The phase separation process is divided into initial and late stages. The smaller the interface thickness $\epsilon$, the further advanced the interface formation, and the shorter the time moving to the late process dynamics. Gameiro et al. [12] investigated numerically the effect of random forces in connection with macrophase separation, and they found that the application of a random force that is almost equal to or greater than the parameter $\epsilon$ smoothes the transition from the initial to the late processes. Because the interface has grown enough so that the domain size is sufficiently larger than the random force in the late process, the random force plays only a secondary role in the global structure. By applying a theory along the lines of coarse 
graining for the interface movement in the late macrophase separation process [18], we found that the domain size growth rule is $\mathcal{P}(t) \propto t^{1 / 3}$. Recall that the Euler characteristic becomes $\chi(\Gamma(\bar{u}))=\int d S K / 2 \pi \propto \mathcal{P}^{-3} \propto t^{-1}$, based on the curvature $\kappa \propto \mathcal{P}^{-2}$, and the surface area $S(\Gamma(\bar{u}))=\operatorname{Per}\left(u^{-}, \Omega\right) \propto \mathcal{P}^{-1}$, from simple dimensional analysis [2]. Further, the Betti numbers, $\beta_{0}\left(X^{ \pm}\right)$and $\beta_{2}\left(X^{ \pm}\right)$, are $O\left(10^{2}\right)$, while $\beta_{1}\left(X^{ \pm}\right)$, which indicates the number of tunnels, is $O\left(10^{4}\right)$; therefore, it is apparent that a similar $t^{-1}$ law also holds for the first Betti number $\beta_{1}\left(X^{ \pm}\right) \simeq-\chi\left(X^{ \pm}\right)=-\chi(\Gamma(\bar{u})) / 2 \propto t^{-1}$.

The difference between macro- and microphase separation dynamics appears in the late process, in which the interface develops. In this stage, the effect of the nonlocal term $\mathcal{E}_{3}$ emerges, resulting in equal contributions from each of the energy terms and restrained domain growth in microphase separation. The difference between the macro- and microphases arises around the time the Betti number reaches $\beta_{1}\left(X^{ \pm}\right) \sim$ $O\left(10^{2}\right)$, regardless of the parameter $\epsilon$. The time corresponds to the emergence of a huge domain that has grown enough to inform us of the boundary $\partial \Omega$. If this occurs before the interface is fully developed, the domain network will undergo a large-scale rearrangement toward a more stable lower-energy state, within the limited arrangement of the region $\Omega$. During this process, the Betti number $\beta_{1}$ does not exclusively exhibit a monotonic decrease. In macrophase separation, the flat interface reaches only one final state, but in microphase separation, the countless number of local minimizers remains constant as $\epsilon \rightarrow 0$

4.2 Topological characterization of transition dynamics between periodic morphologies

Our purpose now is to identify the morphological transition dynamics among the ordered morphologies. As a first step, we check the transition from unstable cylinders to stable spheres, at $(\bar{u}, \epsilon)=\left(0.30,4.0 \times 10^{-2}\right)$. In this process, we fix the cell side length, $l \simeq 1.17$, that numerically provides the global minimizers at the phase boundary between cylinders and spheres. As shown in Fig.6(a), the number of hexagonal cylindrical domains is $\beta_{0}\left(X_{\mathrm{C}}^{-}\right)=4$, while the number of bubbles is $\beta_{0}\left(X_{\mathrm{S}}^{-}\right)=16$. For the topological identification of morphology transitions, it is more natural to consider the homology group of $X_{\mathrm{C}}^{-}$relative to $X_{\mathrm{S}}^{-}$. The relative homology $H_{k}\left(X_{\mathrm{C}}^{-}, X_{\mathrm{S}}^{-}\right)$is equal to the regular homology of the quotient space $X_{\mathrm{C}}^{-} / X_{\mathrm{S}}^{-}$, i.e., the topology of $X_{\mathrm{C}}^{-}$ modulo $X_{\mathrm{S}}^{-}$. This homology $H_{k}\left(X_{\mathrm{C}}^{-}, X_{\mathrm{S}}^{-}\right)$measures pipes detected by chain complexes whose boundaries lie in $X_{\mathrm{S}}^{-}$. In this manner, the first relative Betti number, i.e., the rank of $H_{1}\left(X_{\mathrm{C}}^{-}, X_{\mathrm{S}}^{-}\right)$, is obtained as $\beta_{1}\left(X_{\mathrm{C}}^{-}, X_{\mathrm{S}}^{-}\right)=16$. This result confirms that four cylindrical domains were destabilized to form narrow necks in their axial directions, and they were divided into 16 spherical domains.

Next, we check the transition from layers to cylinders with the parameters $(\bar{u}, \epsilon)=$ $\left(0.20,4.0 \times 10^{-2}\right)$. Unlike the above case of a cylindrical domain fragmenting into spherical domains, a perforated layers morphology of $\beta_{i}\left(X_{\mathrm{PL}}^{-}\right)=(2,18,0,0)$ appears as an intermediate state during morphology transition. This transition proceed in two stages: from layers to perforated layers, and from perforated layers to cylinders (Fig. 6(b)). The second relative Betti numbers for the first transition is computed as $\beta_{2}\left(X_{\mathrm{L}}^{-}, X_{\mathrm{PL}}^{-}\right)=16$, and the first relative Betti numbers for the second transition is computed as $\beta_{1}\left(X_{\mathrm{PL}}^{-}, X_{\mathrm{C}}^{-}\right)=16$. 


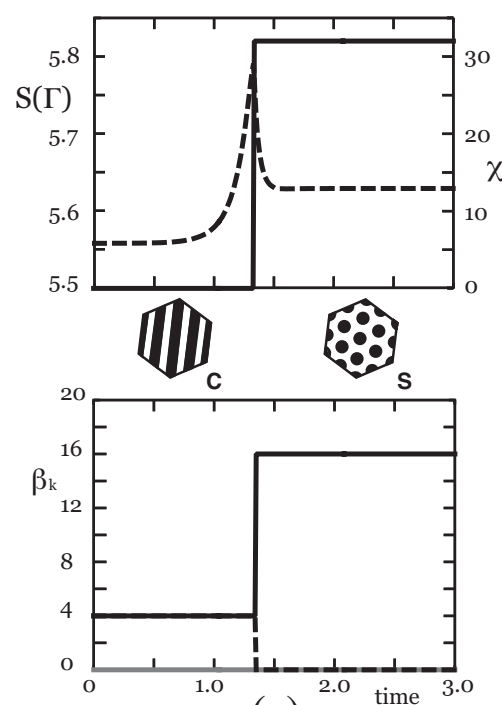

(a)

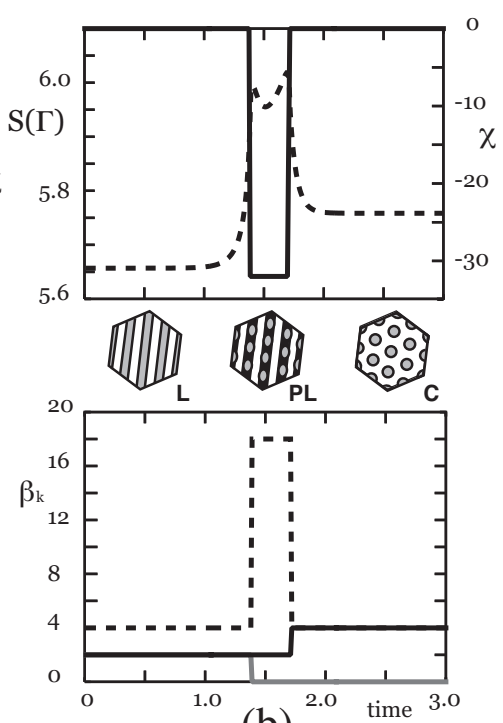

(b)

Fig. 6 (a) Transition from cylinders to spheres for $(\bar{u}, \epsilon)=\left(0.30,4.0 \times 10^{-2}\right)$. Left axis and broken line: surface area $S(\Gamma(\bar{u}))$; right axis and solid line: Euler characteristic $\chi(\Gamma(\bar{u}))$. Solid line: zeroth Betti number $\beta_{0}\left(X^{-}\right)$; broken line: first Betti number $\beta_{1}\left(X^{-}\right)$; gray line: second Betti number $\beta_{2}\left(X^{-}\right)$. (b) Transition from layers to cylinders for $(\bar{u}, \epsilon)=\left(0.20,4.0 \times 10^{-2}\right)$.

Considering cylindrical domains placed in the diagonal direction of the periodic cube region $\Omega$, and assuming $u=-1$ inside the domain, we obtain an energy density $\mathcal{F}_{\epsilon, \sigma}$ at the singular limit as follows:

$$
\frac{\mathcal{F}_{\epsilon, \sigma}}{\epsilon}=\frac{2 \sqrt{2}}{3} \frac{\sqrt{2 \sqrt{3} \pi(1-\bar{u})}}{L}+\left(\frac{\sigma}{\epsilon}\right) \frac{(1-\bar{u})^{2}}{2 \sqrt{3}} L^{2}(c(\bar{u})+R(\zeta)) .
$$

Here, the function $c(\bar{u})$ is defined as the fundamental solution of the Laplace operator; for $R(\zeta)$, see [7]. On the layers-cylinders phase boundary, the interval of hexagonal cylinders alignment $(\sqrt{3} / 2$ times the cylinder center-center distance) is almost equal to $2^{-3 / 2}$ times the laminar stripe interval. Then, the cylindrical domain can be placed inside the laminar domain in the $\langle 110\rangle$ direction in either of the two diagonal directions of the rectangle. Therefore, when the laminar interface is destabilized, eight holes are created on the mesh at the vertical angle $\cos ^{-1}(1 / 3) \approx 70.53^{\circ}$, in this position at which waves in two directions reinforce each other, i.e., perforated layers morphology is formed as an intermediate state. Therefore, during the first stage of transition from layers to cylinders, eight holes are created on each laminar domain and each perforated domain separates into two cylindrical domains in the next stage. Finally, we show the abstract homology generators for the morphologies observed during the transition from layers to cylinders. As shown in Figs.7(a)-(c), the generators for the layers are represented by two-dimensional elementary cubes, and those for the perforated layers and cylinders are represented by one-dimensional cubes. The homology generators are the basis elements for the homology group $H_{k}$, i.e., they are the chain complexes that generate $H_{k}$. In this way, they can be understood as combinations of elementary cubes. The relative 


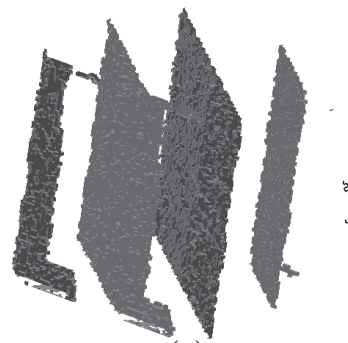

(a)

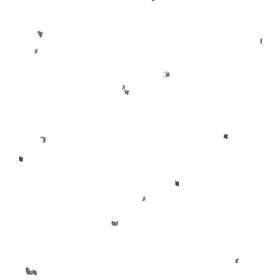

(d)

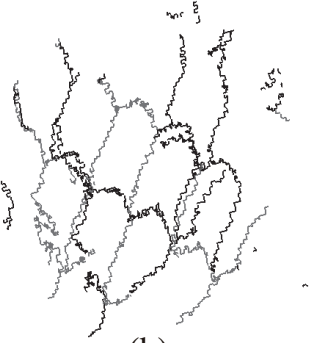

(b)

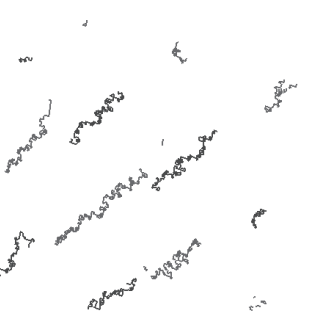

(c)

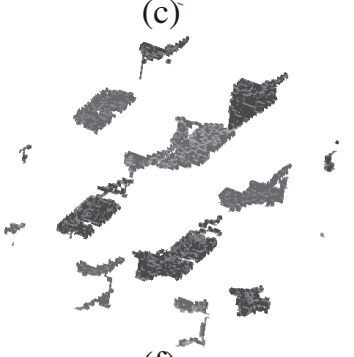

(f)

Fig. 7 Abstract homology generators are shown in (a) Layers; (b) Perforated layers; (c) Cylinders; (d) Layers relative to perforated layers; (e) Perforated layers relative to cylinders; (f) Layers relative to cylinders with $\beta_{2}\left(X_{\mathrm{L}}^{-}, X_{\mathrm{C}}^{-}\right)=\beta_{1}\left(X_{\mathrm{L}}^{-}, X_{\mathrm{C}}^{-}\right)=4$.

cubical sets for $H_{2}\left(X_{\mathrm{L}}^{-}, X_{\mathrm{PL}}^{-}\right)$and $H_{1}\left(X_{\mathrm{PL}}^{-}, X_{\mathrm{C}}^{-}\right)$are also depicted in terms of related cubical sets in Figs.7(d)(e). It is worth noting that if the $k$-th relative Betti numbers provide the number of $k$-dimensional critical events during the morphology transition, a set of generators allows them to be located. At the first transition from layers to perforated layers, perforation holes are created thereby forming a body-centered cubic structure. The relative homology generators during the subsequent transition show the same symmetry, as the perforated layer domains split into cylindrical domains. The morphology transitions are guided by the stable and unstable manifolds of the perforated layers. The perforation (respectively, splitting) can be regarded as a sign of a stable (respectively, unstable) manifold of perforated layer morphology.

\section{Summary}

The microphase separation of diblock copolymer melts has become an excellent model system for studying the fundamental phenomena associated with molecular self-assembly. The composition of the diblock controls the geometry of the morphology. Layers occur in nearly symmetric diblocks. With higher asymmetries, the minority component forms cylinders and spheres. From a geometer's viewpoint, the surface energy plays a key role in determining the morphology that is observed in phase separation. We consider the diblock copolymer problem in the framework of the free-energy functional of a non-local type, and focus on the morphological analysis of energy minimizers in three-dimensional space. We have numerically confirmed that the "balanced scaling law" (i.e., a morphology is attained where two competing terms are balanced $[19,20]$ ) is valid for the singular limit situation, using comparisons with theoretical studies in a 
lower-dimensional space. This view allows us to demonstrate the mechanism behind the appearance of the double gyroid minimizers, by investigating geometric measures such as perimeter and curvatures. Of the candidate morphologies, the double gyroid can obtain the smallest perimeter. In addition, we discuss the geometry of the orthorhombic morphology obtained from our simulations.

These morphologies appear as nodal domains of real-valued functions, i.e., concentration fields of two different blocks. In Section 4, we introduced homological quantities as a measure of the complexity of numerically simulated three-dimensional morphologies. Moreover, we applied computational homology to characterize the complex morphology during phase transition dynamics. The set of the Betti numbers $\beta_{k}$ for a sphere and a cylinder are $(1,0,0,0)$ and $(1,1,0,0)$, respectively. As illustrated in Fig.1, the morphologies of diblock copolymers are lined up by increasing the diblock composition. This indicates that the incremental rule for the Betti numbers would exist in the morphology sequence. The Betti numbers change gradually by adding one along the line-up, so that a perforated layer of $(1,2,0,0)$ is characterized topologically as the intermediate state between a layer of $(1,2,1,0)$ and a cylinder of $(1,1,0,0)$. In fact, the Betti numbers of layer and cylinder relative to a perforated layer are $\beta_{k}\left(X_{L}^{-}, X_{P L}^{-}\right)=(0,0,1,0)$ and $\beta_{k}\left(X_{P L}^{-}, X_{C}^{-}\right)=(0,1,0,0)$, respectively. In addition, the Betti numbers of a bicontinuous morphology of $(1,3,0,0)$ relative to a perforated layer is $\beta_{k}\left(X_{B}^{-}, X_{P L}^{-}\right)=(0,1,0,0)$. In this way, the increment and decrement of the Betti numbers can be associated with the relative Betti numbers, and the corresponding homology generators imply the destinations of the transformation, i.e., the geometry of manifolds connected to a perforated layer. Actually, as shown in the previous section, our topological characterization indicates that the transient perforated layers morphology arises during the layers-cylinders morphology transition. Hence, the perforated layer controls the kinetic pathway during the morphology transition for nearly symmetric compositions. In a three-dimensional space, the perforated layer and bicontinuous morphologies create a zoo of the morphology complex for the diblock copolymer problem.

In this paper, we established a new viewpoint for understanding complex morphology transition dynamics. We incorporated topologically invariant quantities, the so-called homological quantities such as the number of domains and the number of holes, into the field of materials science, and used them as a means of observation. Although the quantities we handle here, such as the Betti number of a polyhedron, are basic and have been known for many years, these items are specifically suited for use in materials science. Nevertheless, we would be very glad if readers gained a sense that the distance between mathematics and materials science is diminishing closer, with computers facilitating the interface.

Acknowledgements The authors would like to thank Rustum Choksi and anonymous referees for valuable comments on the paper. We also thank Ryo Kobayashi and Marcio Gameiro for their help. This work was partially supported by the Grant-in-Aid for Scientific Research 19654015 and the Japanese Ministry of Education, Science, Sports and Culture, Grant-in-Aid for Young Scientists (B)18740238.

\section{References}

1. A Collection Papers of T. Hashimoto: Ordered Structures, Order-Disorder Transition, and Physical Properties of Block Copolymers (1995), Dept. of Polym. Chem., Kyoto University. 
2. Aksimentiev, A., Fiałkowski, M., and Hołyst, R., "Morphology of surfaces in polymer, surfactant, electron and reaction-diffusion systems: methods, measurements and simulations", Adv. Chem. Phys. 121 (2002) 143-239.

3. Bahiana, M., and Oono, Y., "Cell dynamical system approach to block copolymers", Phys. Rev. A 41 (1990) 6763 - 6771.

4. Bailey, T., S., Hardy, C. M., Epps, III, T. H., and Bates, F. S., "A Noncubic Triply Periodic Network Morphology in Poly(isoprene-b-styrene-b-ethylene oxide) Triblock Copolymers", Macromolecules 35 (2002) 7007-7017.

5. Bates, F. S., and Fredrickson, G. H., Block Copolymers - Designer Soft Materials, Physics Today, 52-2(1999), 32-38.

6. Braides, A., $\Gamma$-convergence for Beginners, Oxford University Press (2002).

7. Chen, X., and Oshita Y., Applications of modular functions to interfacial dynamics, Arch. Rat. Mech. Anal. 186 (2007), 109-132.

8. Choksi, R., and Ren, X., "On the Derivation of a Density Functional Theory for Microphase Separation of Diblock Copolymers", J. Stat. Phys. 113 (2003) 151-176.

9. Choksi, R., and Sternberg, P., "Periodic phase separation: the periodic Cahn-Hilliard and isoperimetric problem", Interfaces and Free Boundaries 8 (2006), 371-392.

10. Computational Homology and its Applications, Hokkaido University Technical Report Series in Mathematics, No.124 (2007) [in Japanese].

11. Eyre, D., J., Unconditionally gradient stable time marching the Cahn-Hilliard equation in Computational and Mathematical Models of Microstructual Evolution, (eds. J. W. Bullard, R. Kalita, M. Stoneham, L.-Q. Chen), (MRS, 1998).

12. Gameiro, M., Mischaikow, K., and Wanner, T., Evolution of pattern complexity in the Cahn-Hilliard theory of phase separation, Acta Materialia, 53(2005) 693-704.

13. Goźdź, W. T., and Hołyst, R., "Triply periodic surfaces and multiply continuous structures from the Landau model of microemulsions", Phys. Rev. E 54 (1996) 5012-5027.

14. Grosse-Brauckmann, K., "On gyroid interfaces", J. Colloid Interface Sci. 187 (1997) 418428.

15. Hajduk, D. A., Harper, P. E., Gruner, S. M., Honeker, C. C., Kim, G., and Thomas, E., L., The Gyroid: A New Equilibrium Morphology in Weakly Segregated Diblock Copolymers, Macromolecules 27 (1994) 4063-4075.

16. Kaczynski, T., Mischaikow, K., and Mrozek, M., Computational Homology, SpringerVerlag, NewYork (2004).

17. Kang, X., and Ren, X., "Ring pattern solutions of a free boundary problem in diblock copolymer morphology", Physica D 238 (2009) 645-665.

18. Langer, J. S., An introduction to the kinetics of first-order phase transitions, Solids far from Equilibrium (edited by G. Godreche), Cambridge Univ. Press, Cambridge, 1992.

19. Nishiura, Y., "Far-from-equilibrium dynamics", Translations of Mathematical Monographs Vol. 209, AMS(2002).

20. Nishiura, Y., and Ohnishi, I., "Some mathematical aspects of the micro-phase separation in diblock copolymers", Physica D 84 (1995) 31-39.

21. Nonomura, M., Yamada, K., and Ohta, T., "Formation and stability of double gyroid in microphase-separated diblock copolymers", J. Phys. Condens. Matter 15 (2003) L423-L430.

22. Ohta, T., and Kawasaki, T., "Equilibrium Morphology of Block Copolymer Melts", Macromolecules 19 (1986) 2621-2632.

23. Ren, X., and Wei, J., "On the multiplicity of two nonlocal variational problems", SIAM J. Math. Anal. 31 (2000) 909-924.

24. Spivak, M., A Comprehensive Introduction to Differential Geometry, Publish or Perish, Berkley (1979).

25. Teramoto, T., "Nosé thermostat for the pattern formation dynamics", Mol. Sim. 33 (2007) 71-75.

26. Teramoto, T., and Nishiura, Y., "Double Gyroid Morphology in a Gradient System with Nonlocal Effects", J. Phys. Soc. Jpn. 71 (2002) 1611-1614.

27. Thomas, E. L, Anderson, D. M., Henkee, C. S., and Hoffman, D., "Periodic areaminimizing surfaces in block copolymers", Nature 334 (1988) 598-601. 